\title{
LEITURA GEOGRÁFICA NO CONTEXTO DA COVID-19 EM RONDÔNIA AO NORTE DO
} BRASIL

\section{Geographical reading in the context of COVID-19 in Rondônia to the North of Brazil}

\author{
Laila Cíntia Mota Belforte \\ Mestranda do Programa de Pós-Graduação em Geografia da Universidade Federal de Rondônia/ Bolsista \\ CAPES \\ lailabelforte@gmail.com \\ Rafaela da Silva Pereira Reis \\ Bacharela em Geografia pela Universidade Federal de Rondônia \\ rafaelareis1986@gmail.com \\ Gilberto Paulino da Silva \\ Mestre em Geografia pela Universidade Federal de Rondônia \\ gilberto.paulino@ifro.edu.br \\ Maria Madalena de Aguiar Cavalcante \\ Professora do Departamento de Geografia e Programa de Pós-Graduação da Universidade Federal de \\ Rondônia \\ mada.geoplan@gmail.com
} Artigo enviado para publicação em 21/04/2020 e aceito em 30/04/2020

DOI: $10.12957 /$ tamoios.2020.50379

\section{Resumo}

Estudos em geografia têm desempenhado um papel importante na leitura sobre o avanço na luta contra a COVID-19. A Geografia evidencia a necessidade de identificar, monitorar e reconhcer esse avanço a partir da análise espaçotemporal resultando em subsídio as tomadas de decisões ao considerar os níveis de contaminação alarmante, não só pela letalidade da doença, mas pela possibilidade das esferas governamentais agirem no momento certo e preciso em que determinada área espacial merece maior atenção. Assim, o objetivo do artigo é espacializar os casos da Covid-19 e os números de Unidade de Tratamento Intensivo (UTI) no estado de Rondônia, de modo a fomentar medidas de isolamento social. A metodologia teve como foco a pesquisa bibliográfica e documental e o uso do software Quantum Gis 12.2. Como resultado, verificou-se que os mapas constituem um elemento essencial para a compreensão e visualização da propagação do coronavírus, e a velocidade temporal com que se expande, fato, que tem contribuído enquanto suporte para as decisões sobre a flexibilização ou isolamento social, horizontal. A contribuição para o planejamento, é demonstrado a partir das observações feitas sobre o estado de Rondônia, ao considerar a variável número de UTIs das regiões de saúde (unidade utilizada pelos serviços de saúde) e a portaria 1.101/2002 do Ministério da Saúde no Brasil, revogada pela de $\mathrm{n}^{\circ} 1.631$ de 2015 onde a mesma recomenda sobre a quantidade de leitos que devem cobrir cada 1 mil habitantes, o que não corresponde ao mínimo de 1,5 leitos recomendados, o que enfaticamente aponta o isolamento social como medida eficaz ao enfrentamento da contaminação pelo coronavírus.

Palavras-chave: Geografia; Rondônia; Novo Coronavírus; Isolamento Social.

\begin{abstract}
Studies in geography have played an important role in reading about the progress in the fight against COVID-19. Geography highlights the need to identify, monitor and recognize this advance from the spatio-temporal analysis, resulting in support for decision-making when considering the levels of contamination alarming, not only due to the lethality of the disease, but also due to the possibility of governmental spheres acting on right and precise moment when a given space area deserves more attention. Thus, the objective of the article is to spatialize the cases of Covid19 and the numbers of the Intensive Care Unit (ICU) in the state of Rondônia, in order to promote measures of social isolation. The methodology focused on bibliographic and documentary research and the use of Quantum Gis 12.2 software. As a result, it was found that maps are an essential element for understanding and visualizing the spread of the coronavirus, and the temporal speed with which it expands, a fact that has contributed as a support for decisions about flexibility or social isolation, horizontal. The contribution to planning is demonstrated by the observations made on the state of Rondônia, considering the variable number of ICUs in the health regions (unit used by the health services) and the Ordinance 1,101/ 2002 of the Ministry of Health in Brazil , repealed by No. 1,631 of 2015, where it recommends the number of beds that should cover each 1 thousand inhabitants, which does not correspond to the minimum of 1.5 recommended beds, which emphatically points to social isolation as an effective measure to cope contamination by coronavirus.
\end{abstract}

Key words: Geography; Rondônia; New Coronavirus; Social Isolation. 


\section{Introdução}

O novo agente do coronavírus foi descoberto no final do mês de dezembro (31/12/2019), após casos registrados na China com a doença chamada de COVID-19. Wuhan, cidade chinesa chamou a atenção pela quantidade de casos de uma pneumonia desconhecida, sendo notificada à Organização Mundial de Saúde (OMS). O primeiro óbito em virtude do novo vírus ocorreu em 11 de janeiro de 2020, desde então, o vírus se alastrou pelo mundo, o que levou a OMS a declarar como pandemia no dia 11 de março de 2020 a COVID-19 (OPAS, Brasil, 2020).

Segundo a OMS, até o dia 17 de abril de 2020, foram registrados 150.142 casos de mortes no mundo decorrente da COVID-19 foram contabilizados 2.207 .730 casos em 193 países ou territórios. O número de diagnosticados possivelmente reflete apenas uma parte dos contaminados com o novo Coronavírus, devido às políticas díspares dos diferentes países para a realização dos testes, alguns só os fazem em casos graves quando pessoas precisam de hospitalização. (OPAS, Brasil, 2020).

No Brasil, já são 33.682 casos e 2.141 mortes até o dia 17 de abril de 2020, confirmados pelo Ministério da Saúde. A curva ascendente que registra os casos confirmados não deixa dúvida sobre o que se anuncia, mas também explica a dinâmica das ações de disseminação do vírus no espaço ao longo do tempo.

A Ciência Geográfica, por meio da categoria de análise espaço-tempo, auxilia na compreensão dos fenômenos ocorridos no espaço geográfico ao longo do tempo, que somadas ao uso de ferramentas e as tecnologias como as dos Sistemas de Informação Geográfica (GIS), são instrumentos importantes para monitorar o contexto de disseminação da COVID-19, evidencia quais áreas requer atenção e medidas preventivas.

Os primeiros casos da Covid-19 no estado de Rondônia foram registrados nas cidades que ficam ao longo do eixo da BR 364, principal rodovia interestadual, o que confirma que a evolução dos casos tem relação com o fluxo de pessoas na propagação do vírus (CAVALCANTE, 2020). Os dados consolidados até o dia 15 de abril para o estado de Rondônia registraram 73 casos confirmados, cuja distribuição ratifica o que fora apresentado pela autora, no qual 52 casos estão centrados na capital Porto Velho, 7 no município de Ariquemes, 6 em Ouro Preto do Oeste, 3 em Ji-Paraná, 3 em Rolim de Moura, 1 em Jaru e 1 em Vilhena, o que confirma a maior incidência no número de casos nas cidades destaques com maior densidade demográfica do estado.

Neste contexto, o objetivo do artigo consiste em demonstrar por meio da análise espaço-tempo a importância da ciência geográfica aliado a outras ciências no enfrentamento do coronavírus no estado de Rondônia, agregando a essa discussão a rede assistencial de saúde referente aos leitos de internação complementares por região de saúde, conforme dados do departamento de informática do Sistema Único de Saúde - SUS do Brasil.

\section{A geografia na Análise Espaço-Tempo}

$\mathrm{Na}$ busca pela individualização da geografia enquanto ciência, essa chegou a ser categorizada exclusivamente como ciência de síntese, na compreensão dos fenômenos da superfície da Terra. No entanto, o pensamento de Santos (2012) contribui quando entende a capacidade de síntese não como um privilégio exclusivo de um especialista, mas enquanto resultado da capacitação intelectual que ultrapassa o conhecimento tangente da ciência de formação, assim, alcançando o universo das coisas e a compreensão de cada coisa como um universo.

A geografia é evidenciada em Santos (2012) uma ciência que transita em outras áreas como a demografia e economia entre outras, com conhecimento de causa para a 
materialização de seus objetivos característicos da geografia moderna que é a preocupação em explicar os fenómenos que se propagam no tempo e como se organizam espacialmente . Segundo Santos (2002, p. 48):

Seu objetivo não é explicitamente uma descoberta da experiência humana total, mas, antes da experiência técnica ou da utilização racional do espaço-tempo, visando assegurar eficácia econômica a administração dos investimentos. Todavia, o objetivo correto não é uma geografia preocupada com investimentos, mas com todas as formas de existência.

A geografia tem contribuído com o processo de análise espacial do fenômeno, no caso da espacialização da COVID-19. Cavalcante (2020) corrobora quando diz que a Ciência Geográfica pode contribuir na análise dos fenômenos sociais e naturais, além de agregar-se a outras áreas do conhecimento, principalmente neste momento de crise, revelando a importância do monitoramento detalhado para o enfrentamento da COVID19, especialmente quando se acompanha os números de UTIs por regiões de saúde no estado, colaborando de forma prática para o direcionamento das ações nas esferas governamentais ou onde o isolamento deverá ser a principal medida para quebra das redes de contágio.

\section{Procedimentos metodológicos}

Para análise espaço-temporal da COVID-19 em Rondônia, utilizou-se da pesquisa bibliográfica e documental. Os dados sobre o coronavírus foram extraídos dos boletins oficiais da Agência Estadual de Vigilância em Saúde de Rondônia, os dados populacionais são do Instituto Brasileiro de Gografia e Estatística - IBGE (2010; 2019); bem como os dados de leitos complementares e UTI do Departamento de Informática do Sistema Único de Saúde do Brasil - DATASUS (2020) e do Cadastro Nacional de Estabelecimentos de Saúde - CNES.

Após extração dos dados no DATASUS os mesmos foram sistematizados em gráficos e mapas, representados por regiões de saúde, conforme a Lei 8.080 de 19 de setembro de 1990 e as ações do SUS, as quais são regionalizadas e hierarquizadas chamados de regiões de saúde, conforme definição do Decreto $\mathrm{n}^{\circ} 7.508 / 2011$ como sendo:

Espaço geográfico contínuo construído por agrupamentos de municípios limítrofes, delimitados a partir de identidades culturais, econômicas e sociais e de redes de comunicação e infraestrutura de transportes compartilhados, com a finalidade de integrar a organização, o planejamento e a execução de ações e serviços de saúde (BRASIL, 2011).

Todos os estados do Brasil estão organizados em regiões de saúde, ou seja, grupo de municípios para organização das ações em níveis de complexidade crescente. Os dados de distribuição dos leitos complementares em Rondônia, correspondem ao atendimento de paciente que necessita de assistência especializada, exigindo aspectos especiais como: unidade de isolamento, isolamento reverso, unidade de terapia intensiva e semi-intensiva (VIEGAS, 2016).

Do quantitativo de leitos complementares, os leitos de UTI foram separados, pois a COVID-19 atinge o sistema respiratório, no qual a vítima em estado grave ou crítico necessita de ventilação mecânica, ou seja, possui critério de elegibilidade para admissão em UTI (Portaria de Consolidação n ${ }^{\circ}$ 3/gm/ms/2017).

Com os números de leitos organizados em planilhas, multiplicados por $1.000 \mathrm{e}$ 
100.000 dividindo pelo número da população residente para assim verificar o recomendado pela portaria 1.101/2002 e sua revogação $n^{\circ}$ 1631/GM/MS/2015 conforme propõe Ministério da Saúde no Brasil.

Para a elaboração dos mapas foi utilizado o Software Quantum Gis 2.12 por meio de dados vetoriais, obtidos pelas instituições - IBGE (2015), Ministério da Saúde (2020), Sirgas 2000, Fuso 20s, EPSG: 31980 Datum.

\section{A COVID-19 em Rondônia e os desafios da saúde nos municípios}

Rondônia está localizado na região Norte do Brasil e tem como limites os estados do Mato Grosso, Amazonas, Acre e Bolívia. Possui 52 municípios e ocupa uma área de 237.765,293 km², a rodovia BR-364 é a porta de entrada da Amazônia brasileira.

Diante do quadro de pandemia, o coronavírus chega em Rondônia, conforme noticiado pela Agência Estadual de Vigilância em Saúde (AGEVISA) e a Secretaria de Estado da Saúde (SESAU), por meio dos boletins diários, o que tornou possível espacializar os casos confirmados e de óbitos.

Na primeira representação cartográfica, até o dia 16 de março não havia casos confirmados para COVID-19 em Rondônia (ver figura 01), no entanto, foram registradas 36 notificações com 24 casos aguardando resultado para coronavírus nos municípios a saber: 8 em Ariquemes, 13 casos em Porto Velho, 2 casos em Vilhena e 1 em Ji-Paraná.

A segunda representação cartográfica (ver figura 01) trata-se dos casos confirmados para COVID-19 em Rondônia registrado até o dia 15 de abril de 2020, distribuídos nos seguintes municípios: 52 casos confirmados em Porto Velho, 7 em Ariquemes, 6 em Ouro Preto do Oeste, 3 em Ji-Paraná, 3 em Rolim de Moura, 1 em Jaru e 1 em Vilhena, 2 óbitos em Porto Velho, capital do estado, e 1 em Ji-Paraná.

O mapa confirma a constatação de Cavalcante (2020), haja vista que a disseminação da doença se configura nas cidades localizadas nas margens da BR-364, cidades que se caracterizam por sua importância econômica no estado. Os dados indicam que os municípios que são contíguos a BR-364, tais como Porto Velho, Ariquemes, Rolim de Moura, Ouro Preto do Oeste, Ji- Paraná, Jaru, Vilhena, confirmam a tendência de disseminação pelo principal fluxo rodoviário do estado, sendo a principal via que interliga Rondônia às demais regiões Norte e Centro-Oeste do país.

Figura 01 - Mapa de disseminação da COVID-19 por municípios em Rondônia.

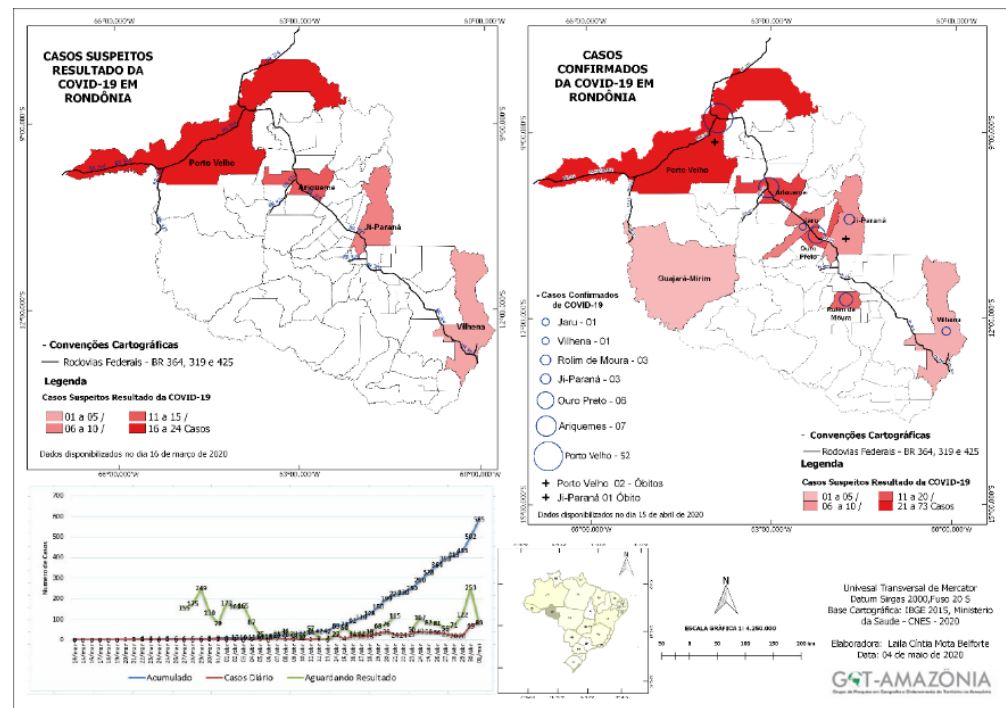

Fonte: Elaborado pelas autoras (2020). 
A leitura geográfica do avanço da COVID-19 no território Rondoniano e seus arranjos como rodovias, densidade demográfica e equipamentos de saúde, contribui enquanto indicativos em relação às medidas colaborativas ao combate do coronavírus, visto que o isolamento social é o mais indicado no Brasil e no mundo. Neste sentido, a leitura do território a partir da cartografia permite compreender as principais regiões de contágio, para então obter uma maior adesão aos isolamentos por meio da informação e das campanhas de conscientização que precisam ser intensificadas.

O percentual de isolamento social em Rondônia em 01 de maio de 2020, foi de $33,7 \%$ conforme demonstra o Gráfico 01 . O Índice de Isolamento Social (IIS), por estado, é um termômetro que por meio de tecnologia ${ }^{1}$ de localização de $60 \mathrm{M}$ de brasileiros, auxilia a compreender em que medida a população está respeitando a recomendação de isolamento.

Gráfico 01 - Percentual de Isolamento social em Rondônia.

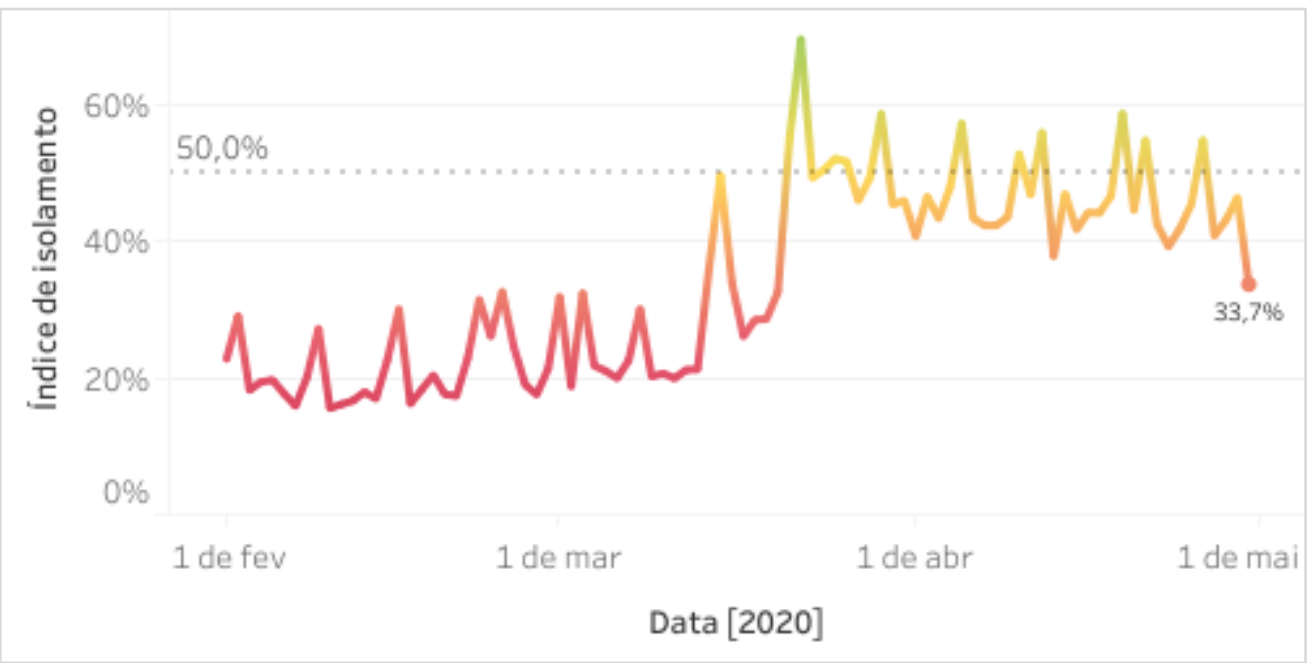

Fonte: Dados de Índice de Isolamento Social (In loco - 2020).

De acordo com o centro de contingência à adesão, o índice ideal é de $70 \%$. Deste modo, se a taxa continuar baixa, o número de leitos disponíveis no Sistema de Saúde em Rondônia não será o suficiente para atender a população em poucos dias. As internações iniciaram no dia 15 de abril de 2020, com o registro de 04 pacientes internados com COVID-19, 02 na cidade de Porto Velho (Centro de Medicina Tropical de Rondônia/público e hospital Central/particular); 01 em Cacoal no hospital regional; e 01 em Ji-Paraná no hospital municipal.

Embora, as internações sejam ainda em pequeno número em relação a outros estados como São Paulo, Ceará entre outros, é preciso destacar que a COVID-19 em Rondônia é mais recente, e considerando a realidade dos equipamentos de saúde, sobretudo quando é revelado o número de leitos de UTI para atender sua população, verifica-se que a infraestrutura disponível está aquém das demandas rotineiras, principalmente ao se observar a situação no interior do estado. Os municípios estão desprovidos para receber um número elevado de pessoas em UTIs, como demonstra a Figura 02 sobre a espacialização de número de leitos. Há ainda o agravante do baixíssimo estoque de testes, sendo feito apenas em casos graves, o que torna o número de casos confirmados inferior aos números divulgados oficialmente. 
Figura 02 - Espacialização do número de Leitos complementares e UTI’s por região de saúde no Estado de Rondônia.

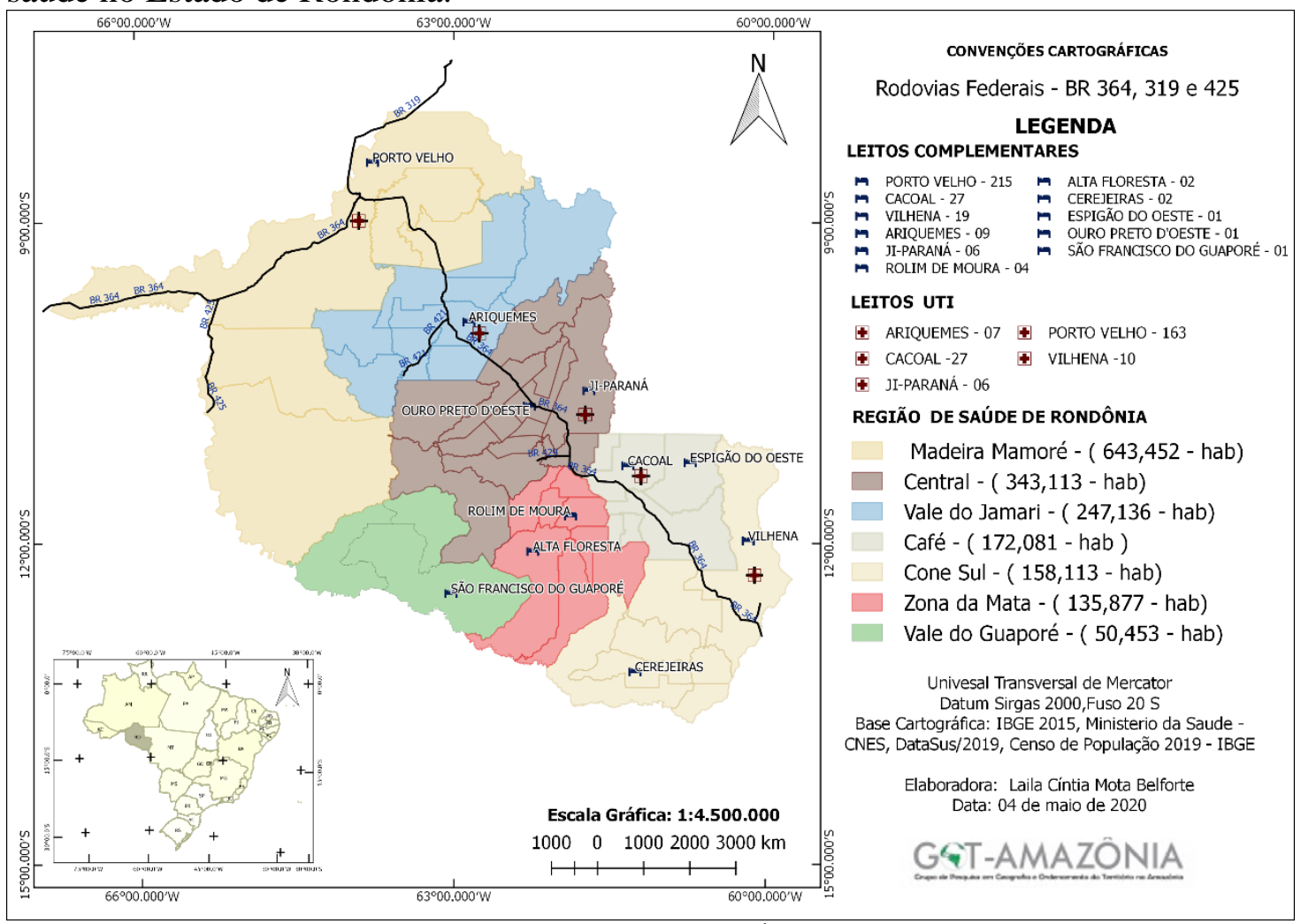

Fonte: Elaborado pelas autoras (2020) a partir do Sistema Único de Saúde registrados em fevereiro de 2020.

A cartografia, como pode ser observado, permite uma leitura didática sobre o quantitativo correspondente as UTI's por regiões de saúde e ao considerar que o maior número de casos da COVID-19 tendem a concentrar nos municípios que possuem ligação direta com a BR-364, a atenção sobre a infraestrutura para aplicação das ações do SUS torna-se mais clara.

Segundo o DATASUS, o estado de Rondônia conta com o total de 287 leitos complementares e 213 UTIs e ao observar sua distribuição espacial por regiões de saúde, a região central do estado, é uma das mais preocupantes, pois, possui um menor número de leitos em relação as demais, esta região é composta por 14 municípios, onde 4 deles estão localizados no eixo da BR 364, sendo: Ji-Paraná, Ouro Preto do Oeste, Jaru e Presidente Médici. Para esta região, o isolamento social apresenta-se como medida essencial para evitar que os serviços de saúde não sejam saturados.

A região de maior assistência é a região Madeira Mamoré, conforme dispõe o gráfico 02 , onde está localizada a capital do estado, e concentra $75 \%$ do número de leitos, sendo os $25 \%$ dos leitos distribuídos nas demais regiões. 
Gráfico 02 - Distribuição dos Leitos Complementares e UTI por Região de Saúde - RO.

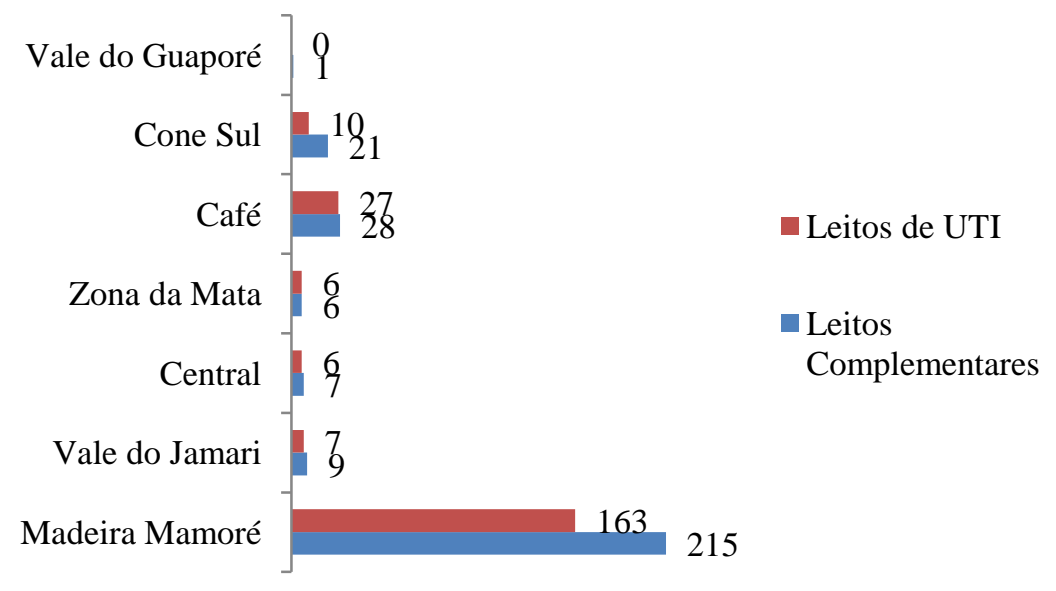

Fonte: DATASUS, dados do mês de fevereiro de 2020.

O Ministério da Saúde estabelece uma média de 1,5 a 3 leitos de $\mathrm{UTI}^{2}$ para cobertura populacional de 1.000 habitantes, o indicado é 18 leitos para cada $100 \mathrm{mil}$ habitantes. Com base nesse critério ao avaliar as regiões de saúde de Rondônia, apenas a região Madeira Mamoré corresponde ao recomendado, as demais em condições normais já não dispõe da média necessária, o que as tornam suscetíveis à crise no atendimento de saúde no enfrentamento da pandemia.

A região crítica no que se refere à disposição de leitos é a do Vale do Guaporé, pois não disponibiliza nenhum leito de UTI, sendo essa região de saúde composta por 3 municípios: São Francisco do Guaporé, Costa Marques e Seringueiras, e ficam a mais de $500 \mathrm{~km}$ de distância das regiões que integra os serviços de saúde de alta complexidade, porém, por estar fora do eixo da principal via de circulação, não tem relação direta com a BR 364, de onde advém o vetor de maior fluxo de pessoas, por conseguinte, de disseminação do vírus. Dependendo da ação da gestão pública, pode ser a região de fácil controle da COVID-19, caso cumpram o isolamento e, principalmente, medidas de controle de acesso na região, vista a precária assistência à saúde.

As análises corroboram com o isolamento horizontal como a melhor medida de controle de disseminação da COVID-19 em Rondônia, haja vista os números disponíveis de leitos, que já deu sinais de preocupação com o fato ocorrido no último dia 14 de abril de 2020, onde uma idosa com suspeita da COVID-19 ao ser transportada por uma UTI móvel, saindo da cidade de Ji-Paraná para o município de Cacoal, acabou falecendo durante o percurso. $\mathrm{O}$ translado ocorreu porque no município de Ji-Paraná, localizado na região Central do estado, possui apenas 6 leitos de UTI, e esses estavam ocupados, fato que levou a paciente ser levada para a região do Café no município de Cacoal (G1 - RO, 2020).

Dos três casos de óbitos registados pela COVID-19 em Rondônia, dois foram de pessoas acima dos 60 anos, fato que afirma a estatística registrada por Geiss (2020), na qual pacientes entre 60-70 anos têm uma probabilidade de 0,4\% de morrer, aqueles com idades entre 70-80 anos têm 1,3\% e os com mais de 80 anos, de 3,6\% . O monitoramento diário, inclusive com os endereços dos casos confirmados e a faixa etária tornam-se ferramentas informacionais importantíssimas para a espacialização e possíveis analogias.

A localização dos casos suspeitos, confirmados e de óbitos podem contribuir para estratégias do retardamento do avanço do coronavírus, uma vez que, medidas restritivas, segundo especialistas, ainda são as melhores alternativas a serem tomada para evitar a 
contaminação e proteger a população nos municípios nos quais o vírus ainda não chegou, ou até mesmo onde o contágio já ocorreu, mas, ainda não se alastrou pelas comunidades, impedindo ou minimizando a sobrecarga do sistema de saúde. A Geografia, deste modo, mostra-se essencial no mapeando e leitura na expansão da COVID-19

\section{Considerações finais}

A geografia na leitura dos fenômenos apresenta arcabouço teórico e metodológico que pode contribuir para o enfrentamento da COVID-19. Exemplo é o da cartografia digital que a partir da leitura do território e seus atributos revela áreas com maior ou menor incidência da pandemia do coronavírus, área cuja estrutura em saúde precisa de investimentos, como aquelas demonstradas no estado de Rondônia, que não correspondem ao mínimo de 1,5 leitos recomendados, com destaque às regiões de saúde Vale do Guaporé e Central.

A atualização das informações e sua veracidade a exemplo dos dados sobre os equipamentos de saúde, população e a regularidade nas publicações dos boletins oficiais da COVID-19 são fundamentais para que, ao serem espacializadas, possam de fato representar a organização territorial ou espacial enquanto instrumento para $o$ planejamento, visto que a utilização correta das informações resultantes do monitoramento ou até mesmo omissão de informação pode revelar-se um dos maiores problemas ao enfrentamento do vírus.

Os dados geográficos contribuem à atuação da gestão pública, fornecendo suportes em vista de evitar que a curva de contaminação não se eleve, retardando a contaminação no espaço de tempo para que o Sistema de Saúde possa atender a demanda. Caso contrário, a propagação da COVID-19 em um espaço curto de tempo colocará o Sistema de Saúde em colapso.

Por fim, a principal barreira para as contribuições da geografia se tornar políticas públicas direcionadas, esta na pressão que as esferas administrativas vêm tendo quanto as medidas adotadas pelos governos de isolamento social ao impactarem a economia.

\section{Notas}

1 - A start up In Loco publica mapa do Brasil com índices de isolamento social baseado na localização de celulares. O mapa mostra o percentual da população que está respeitando a recomendação de isolamento para conter a pandemia da COVID-19.

2 - Ministério da Saúde portaria No 1.631 , de $1^{\circ}$ de outubro de 2015.

\section{Referências bibliográficas}

BRASIL, Ministério da Saúde. PORTARIA DE CONSOLIDAÇÃO No 3/GM/MS, DE 28 DE SETEMBRO DE 2017. https://bvsms.saude.gov.br/bvs/saudelegis/gm/2017/MatrizesConsolidacao/Matriz-3-

Redes.html. Acesso em: 10 de abr. de 2020.

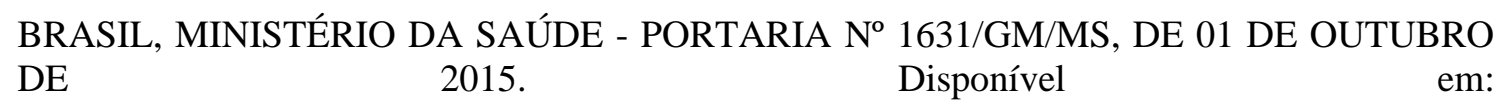
https://bvsms.saude.gov.br/bvs/saudelegis/gm/2015/prt1631 01 10 2015.html. Acesso em: 01 de maio de 2020.

CADASTRO NACIONAL DE ESTABELECIMENTOS DE SAÚDE (CNES) - Disponível em: https://wiki.saude.gov.br/cnes/index.php/P\%C3\%A1gina_principal Acesso em: 10 de abr. de 2020. 
CAVALCANTE, Maria Madalena de Aguiar. Associação Brasileira de Ciência Politíca (ABPC) A Globalização Perversa do COVID-19. Disponível em: https://cienciapolitica.org.br/noticias/2020/04/boletim-9-globalizacao-perversa-covid-19exemplo-rondonia. Acesso em: 09 de abr. de 2020.

G1 - Disponível em: https://g1.globo.com/ro/rondonia/noticia/2020/04/16/prefeitura-confirmaprimeira-morte-por-coronavirus-em-ji-parana-ro.ghtml Acesso em: 19 de abr. de 2020.

FERRAZ, Ligya Helena Valle da Costa. O SUS, o DATASUS e a informação em saúde: uma proposta de gestão participativa. Rio de Janeiro: s.n., 2009.

GEISS, Brian. THE CONVERSATION. Older people are at more risk from COVID-19 because of how the immune system ages. Disponível em: https://theconversation.com/olderpeople-are-at-more-risk-from-covid-19-because-of-how-the-immune-system-ages-133899.

Acesso em: 13 de abr. de 2020.

IBGE - Instituto Brasileiro de Geografia e Estatística. Disponível em: https://cidades.ibge.gov.br/brasil/ro/panorama. Acesso em: 11 de abr. de 2020.

IN LOCO - Média do indice de isolamento por estado. Disponível em: https://www.inloco.com.br/pt/? hstc=753710.63a0faadd82161b5abf42f8a72679e5b.15868902 $72748.1586890272748 .1586890272748 .1 \&$ hssc $=753710.1 .1586890272748 \&$ \&sfp $=299838$ 0158. Acesso em: 01 de maio de 2020.

ORGANIZAÇÃO PAN-AMERICANA DA SAÚDE (OPAS). Disponível em: https://www.paho.org/bra/index.php?option=com_content \&view=article\&id=6101: covid19\&Ite mid=875. Acesso em: 19 de abr. de 2020.

RONDÔNIA. Agência Estadual de Vigilância em Saúde (AGEVISA) e a Secretaria de Estado da Saúde (SESAU), boletins referentes ao coronavírus (Covid-19) no Estado de Rondônia. Disponível em: http://www.rondonia.ro.gov.br/. Acesso em: 10 de abr. de 2020

SANTOS, Milton. Por uma Geografia Nova: Da Crítica da Geografia a uma Geografia Crítica. 6. Ed., $2^{\circ}$. reimpr. - São Paulo: Editora da Universidade de São Paulo, 2012.

SANTOS, Milton. A natureza do Espaço: Técnica e Tempo, Razão e Emoção - $4^{\circ}$ ed. 8 reimpr. - São Paulo: Editora da Universidade de São Paulo, 2014.

SANTOS, Lenir. Região de Saúde e suas Redes de Atenção: modelo organizativo-sistêmico do SUS. Ciência \& Saúde Coletiva, 22 (4): 1281-1289 2017.

UNIVERSIDADE ABERTA DO SUS - UNASUS - Disponível em: https://www.unasus.gov.br/noticia/organizacao-mundial-de-saude-declara-pandemia-decoronavirus. Acesso em: 10 de abr. de 2020.

VIEGAS, Ana Paula Bise et al. Manual de Conceitos e Nomenclatura de Leitos Hospitalares. Empresa Brasileira de Serviços hospitalares. $1^{\circ}$ edição, Agosto de 2016. 